\title{
High processed meat consumption is a risk factor of type 2 diabetes in the Alpha-Tocopherol, Beta-Carotene Cancer Prevention study
}

\author{
Satu Männistö ${ }^{1 *}$, Jukka Kontto ${ }^{1}$, Merja Kataja-Tuomola ${ }^{1}$, Demetrius Albanes ${ }^{2}$ and Jarmo Virtamo ${ }^{1}$ \\ ${ }^{1}$ Department of Chronic Disease Prevention, National Institute for Health and Welfare (formerly National Public Health Institute), \\ PO Box 30, FI-00271 Helsinki, Finland \\ ${ }^{2}$ National Cancer Institute, National Institute of Health, Bethesda, MD, USA
}

(Received 2 June 2009 - Revised 9 December 2009 - Accepted 4 January 2010 - First published online 26 February 2010)

Relatively small lifestyle modifications related to weight reduction, physical activity and diet have been shown to decrease the risk of type 2 diabetes. Connected with diet, low consumption of meat has been suggested as a protective factor of diabetes. The aim of the present study was to examine the association between the consumption of total meat or the specific types of meats and the risk of type 2 diabetes. The Alpha-Tocopherol, Beta-Carotene Cancer Prevention cohort included middle-aged male smokers. Up to 12 years of follow-up, 1098 incident cases of diabetes were diagnosed from 24845 participants through the nationwide register. Food consumption was assessed by a validated FFQ. In the age- and intervention group-adjusted model, high total meat consumption was a risk factor of type 2 diabetes (relative risk (RR) $1.50,95 \%$ CI 1.23, 1.82, highest $v$. lowest quintile). The result was similar after adjustment for environmental factors and foods related to diabetes and meat consumption. The RR of type 2 diabetes was 1.37 for processed meat $(95 \%$ CI $1.11,1.71)$ in the multivariate model. The results were explained more by intakes of $\mathrm{Na}$ than by intakes of SFA, protein, cholesterol, haeme Fe, $\mathrm{Mg}$ and nitrate, and were not modified by obesity. No association was found between red meat, poultry and the risk of type 2 diabetes. In conclusion, reduction of the consumption of processed meat may help prevent the global epidemic of type 2 diabetes. It seems like $\mathrm{Na}$ of processed meat may explain the association.

Cohort studies: Epidemiology: Meat: Processed meat: Diabetes

It has been predicted that the number of adults with diabetes will double during the next two decades, being 300 million worldwide in the year $2025^{(1)}$. Because of the long-term serious complications and indirect mortality of diabetes, all established preventive factors against the disease are valuable. From lifestyle factors, obesity and physical inactivity have consistently been associated with an increased risk of type 2 diabetes ${ }^{(2,3)}$. The intervention studies have also shown the possibility to reduce the risk of type 2 diabetes by relatively small lifestyle modifications in weight control, physical activity and diet ${ }^{(4,5)}$.

Three cohort studies from the United States have shown that high consumption of meat, particularly processed meat, may increase the risk of type 2 diabetes in men ${ }^{(6)}$ and women ${ }^{(7,8)}$. The only cohort study outside the United States found that especially high consumption of processed meat increased the risk of type 2 diabetes among overweight and obese Chinese women ${ }^{(9)}$. The mechanisms behind the observed relationship are unclear.

The prospective data from the Alpha-Tocopherol, BetaCarotene Cancer Prevention study (ATBC study) were used to examine the relationship between the consumption of total meat and specific types of meats (red meat, processed meat and poultry) and the risk of type 2 diabetes in Finnish middle-aged male smokers. Furthermore, the explanatory factors related to meat and whole diet (alcohol, fruits, vegetables, rye, milk, coffee, SFA, protein, cholesterol, haeme $\mathrm{Fe}, \mathrm{Mg}$, $\mathrm{Na}$, nitrate and energy) were examined.

\begin{abstract}
Methods
The ATBC Study was a randomised, double-blinded, placebo-controlled clinical trial undertaken to determine the effects of antioxidant supplements on cancer among male smokers aged 50-69 years and living in Southwestern Finland $\left(n\right.$ 29133) ${ }^{(10,11)}$. At baseline, men were excluded if they smoked fewer than five cigarettes a day and had a previous history of cancer, severe angina on exertion, chronic renal insufficiency, liver cirrhosis, alcoholism or other medical conditions limiting long-term participation. Furthermore, men who received anticoagulant therapy or used vitamin $\mathrm{E}$, vitamin A or $\beta$-carotene supplements in excess of predefined doses were excluded. The recruitment was carried out between 1985 and 1988, and the trial intervention continued until April 1993. The trial cohort had been followed up through national registers thereafter.

The present study was conducted according to the guidelines laid down in the Declaration of Helsinki, and all procedures involving human subjects were approved by the Institutional Review Boards of the National Public Health
\end{abstract}

Abbreviations: ATBC, Alpha-Tocopherol, Beta-Carotene Cancer Prevention; RR, relative risk.

* Corresponding author: Satu Männistö, fax +358 20610 8338, email satu.mannisto@thl.fi 
Institute (currently the National Institute for Health and Welfare), Finland, and the National Cancer Institute, USA. Written informed consent was obtained from all the subjects.

\section{Ascertainment of diabetes}

In Finland, patients needing medical treatment for diabetes are entitled to reimbursement of their medication expenses according to the sickness insurance legislation. This requires a medical certificate from the attending physician. The certificate of every case is verified to fulfil the diagnostic criteria (blood glucose permanently $7.0 \mathrm{mmol} / \mathrm{l}$ or higher after dietary treatment) for diabetes at the Social Insurance Institution which maintains a central register of all the persons receiving drug reimbursement. The participants of the ATBC study were linked to the register through the unique personal identity number assigned to each Finnish citizen.

At baseline, 1272 participants had a history of diabetes diagnosed by a physician. Furthermore, 1918 participants were excluded because of an incompletely filled-in FFQ. After the exclusions, the final cohort for the present study comprised 25943 men, among whom 1098 incident cases of diabetes were identified from the drug reimbursement register through December 1997 (followed up to 12 years).

\section{Baseline data collection}

At baseline, each man completed questionnaires on general characteristics as well as on medical history, smoking and physical activity. Height and weight were measured, and BMI $\left(\mathrm{kg} / \mathrm{m}^{2}\right)$ was calculated. Blood pressure was measured using a mercury sphygmomanometer from the right arm, while the subject remained seated. Serum samples were collected and stored at $-70^{\circ} \mathrm{C}$. Serum glucose was determined by the enzymatic hexokinase method using an Optima analyser (ThermoFischer, Vantaa, Finland). Serum total cholesterol concentrations were determined enzymatically (cholesterol oxidase- $p$-aminophenazone, method; Boehringer Mannheim, Mannheim, Germany). HDL- cholesterol was measured after precipitation with dextran sulphate and $\mathrm{MgCl}_{2}$.

\section{Dietary assessment}

Food consumption over the previous 12 months was assessed at baseline with a validated self-administered FFQ developed for the ATBC study ${ }^{(12)}$. The consumption of 276 food items and mixed dishes (about fifty food items or dishes including meat, sausage or poultry) was recorded by asking the number of times an item was usually consumed per day, week or month. Participants were also allowed to report additional foods consumed frequently but not listed in the FFQ. The portion size was assessed by a picture booklet including 122 colour photographs of food items or dishes. The participants completed the FFQ at home, and returned it during the second baseline visit, where a trained study nurse checked the FFQ thoroughly and modified possible discrepancies during a $30 \mathrm{~min}$ interview. Thereafter, a senior nutritionist reviewed all the FFQ for final approval. In all, $93 \%$ of the FFQ were approved. The food data were converted into daily meat consumption (total meat, red meat (beef and pork), processed meat and poultry) and nutrient intakes according to the software and food composition database at the National Public Health Institute in Finland. We did not assess the fish consumption in the present study.

The reproducibility and validity of the dietary questionnaire were tested in a pilot study with 189 men using a $24 \mathrm{~d}$ food record $(2 \times 12 \mathrm{~d})$ as a reference method ${ }^{(12)}$. For the meat variables, the extended analyses of crude intraclass correlations between the first and second FFQ ranged from 0.56 (pork) to 0.74 (poultry), and the correlation coefficient between the first FFQ and the food records ranged from 0.31 (pork) to 0.50 (processed meat).

\section{Statistical analyses}

The associations between quintiles of meat consumption and the incidence of diabetes were calculated by Cox proportional hazards regression, and are expressed as relative risks (RR) and $95 \%$ CI. The proportional hazards assumption was tested with no evidence of non-proportional hazards. Personyears of follow-up were calculated from the date of randomisation to the date of diabetes occurrence, death or the end of follow-up (December 1997), whichever came first.

The first model (Model 1) was adjusted for age and intervention groups ( $\alpha$-tocopherol, $\beta$-carotene, both or placebo). Model 2 was further adjusted for BMI, number of cigarettes smoked daily, smoking years, systolic blood pressure, diastolic blood pressure, serum total cholesterol and serum HDLcholesterol, leisure-time physical activity and intakes of alcohol and energy. Furthermore, the multivariate Model 3 was adjusted for all the variables included in Model 2 plus consumption of foods related to type 2 diabetes (fruits, vegetables, rye, milk and coffee). We also added (Model 4) intakes of $\mathrm{SFA}$, protein, cholesterol, haeme $\mathrm{Fe}, \mathrm{Mg}, \mathrm{Na}$ and nitrate to examine mechanisms/explanatory factors behind the results (data not shown). Nutrient intakes were adjusted for energy according to the residual method ${ }^{(13)}$.

Tests for linearity of the trend across the categories were performed using the Wald test by modelling the median value of each quintile as a continuous variable.

The likelihood ratio test was used to study whether BMI modified the effect of meat consumption on diabetes incidence.

All analyses were carried out with the $\mathrm{R}$ statistical program version 2.7.2 ( $\mathrm{R}$ Foundation for Statistical Computing, Vienna, Austria) ${ }^{(14)}$. All $P$ values were two sided, and $P<0.05$ was considered statistically significant.

\section{Results}

On average, men with high consumption of total meat were younger, more obese and physically less active and had more energy in their diet compared with the others (Table 1). The consumption of total meat was 3-fold higher in the highest quintile compared with the lowest quintile. Especially, the consumption of processed meat and poultry was relatively high among those in the highest quintile of total meat consumption. Furthermore, men whose diet was rich in meat tended to have a higher intake of other foods and nutrients as well.

The Pearson coefficients of correlation (adjusted for energy) between the consumption of total meat and specific types of 
Table 1. Age-standardised baseline characteristics (medians) by quintiles of total meat consumption among 25943 men in the Alpha-Tocopherol, Beta-Carotene Cancer Prevention study, Finland, 1985-97*

\begin{tabular}{|c|c|c|c|c|c|}
\hline & \multicolumn{5}{|c|}{ Quintiles of total meat intake } \\
\hline & 1 & 2 & 3 & 4 & 5 \\
\hline \multicolumn{6}{|l|}{ Background characteristics } \\
\hline$n$ & 5189 & 5188 & 5189 & 5188 & 5189 \\
\hline Age (years) & 59 & 58 & 57 & 56 & 56 \\
\hline BMI $\left(\mathrm{kg} / \mathrm{m}^{2}\right)$ & $25 \cdot 7$ & $25 \cdot 7$ & $25 \cdot 9$ & $26 \cdot 0$ & $26 \cdot 2$ \\
\hline Cigarettes smoked daily (no.) & 20 & 20 & 20 & 20 & 20 \\
\hline Smoking years & 37 & 36 & 36 & 36 & 37 \\
\hline Systolic blood pressure (mmHg) & 140 & 140 & 140 & 140 & 140 \\
\hline Diastolic blood pressure $(\mathrm{mmHg})$ & 88 & 88 & 88 & 88 & 88 \\
\hline Serum total cholesterol $(\mathrm{mmol} / \mathrm{l})$ & $6 \cdot 12$ & $6 \cdot 16$ & $6 \cdot 18$ & $6 \cdot 23$ & $6 \cdot 19$ \\
\hline Serum HDL- cholesterol (mmol/l) & 1.14 & $1 \cdot 15$ & $1 \cdot 15$ & $1 \cdot 16$ & 1.15 \\
\hline Leisure-time physical activity (\%)† & 59 & 60 & 60 & 58 & 56 \\
\hline Energy $(\mathrm{kJ} / \mathrm{d})$ & 8985 & 10003 & 10836 & 11749 & 13453 \\
\hline Alcohol (g/d) & 8 & 10 & 11 & 12 & 14 \\
\hline Fruits $(\mathrm{g} / \mathrm{d})$ & 138 & 160 & 176 & 184 & 199 \\
\hline Vegetables $(\mathrm{g} / \mathrm{d})$ & 219 & 255 & 282 & 311 & 338 \\
\hline Rye $(g / d)$ & 77 & 80 & 83 & 83 & 87 \\
\hline Milk (g/d) & 466 & 499 & 528 & 543 & 595 \\
\hline Coffee $(g / d)$ & 550 & 550 & 550 & 600 & 600 \\
\hline \multicolumn{6}{|l|}{ Meat-related characteristics } \\
\hline Total meat $(\mathrm{g} / \mathrm{d})$ & 79 & 111 & 139 & 174 & 244 \\
\hline Red meat $(\mathrm{g} / \mathrm{d})$ & 40 & 54 & 63 & 74 & 88 \\
\hline Beef $(g / d)$ & 12 & 18 & 22 & 26 & 30 \\
\hline Pork (g/d) & 25 & 33 & 38 & 44 & 52 \\
\hline Processed meat $(\mathrm{g} / \mathrm{d})$ & 28 & 46 & 62 & 84 & 139 \\
\hline Poultry (g/d) & 2 & 8 & 10 & 14 & 14 \\
\hline SFA $(g / d)$ & 40 & 45 & 49 & 53 & 63 \\
\hline Protein $(\mathrm{g} / \mathrm{d})$ & 74 & 83 & 91 & 100 & 116 \\
\hline Cholesterol (mg/d) & 413 & 478 & 533 & 594 & 719 \\
\hline Haeme Fe (mg/d) & 1.9 & $2 \cdot 6$ & $3 \cdot 1$ & $3 \cdot 7$ & 4.8 \\
\hline $\mathrm{Mg}(\mathrm{mg} / \mathrm{d})$ & 411 & 443 & 465 & 493 & 542 \\
\hline $\mathrm{Na}(\mathrm{mg} / \mathrm{d})$ & 3745 & 4272 & 4723 & 5282 & 6285 \\
\hline Nitrate $(\mathrm{mg} / \mathrm{d})$ & 40 & 48 & 54 & 60 & 66 \\
\hline
\end{tabular}

*All differences were statistically significant, except for diastolic blood pressure and leisure-time physical activity. † Moderate or heavy activity at leisure time.

meats (red meat, processed meat and poultry) were $0 \cdot 48,0 \cdot 82$ and $0 \cdot 27$, respectively. Instead, correlations between the consumption of processed meat and the other types of meats (red meat, beef, pork and poultry) ranged from -0.04 to -0.02 . Total meat, especially pork and processed meat, correlated positively with energy intake $(r>0 \cdot 35)$.

In the model adjusted for age and intervention groups, the RR of type 2 diabetes was significantly higher by $50 \%$ for the highest $v$. the lowest quintile of total meat consumption (Table 2). The association did not change after adjustment for confounding factors related to diabetes (RR 1.45; $95 \%$ CI $1.16,1.81 ; P$ value, test for trend $<0.001)$ and foods (RR 1.50 ; $95 \%$ CI 1.19, 1.89; $P$ value, test for trend $<0.001)$. Among nutrients, the association between total meat consumption and the risk of diabetes was slightly attenuated by an additional adjustment for $\mathrm{Na}$ (RR 1.28; $95 \%$ CI 1.00, 1.64; $P$ value, test for trend $0 \cdot 04$ ).

The RR of type 2 diabetes was 1.46 (95\% CI 1.20, 1.77; $P$ value, test for trend $<0.001)$ for the highest quintile compared with the lowest quintile of processed meat consumption. The association was attenuated slightly after adjustment for confounding factors related to type 2 diabetes (RR 1.35; $95 \%$ CI 1.09, 1.68; $P$ value, test for trend $<0.001)$ and foods (RR 1.37; $95 \%$ CI 1.11, $1.71 ; P$ value, test for trend $=0 \cdot 001$, but it remained statistically significant. The attenuation of RR was explained more by the intakes of $\mathrm{Na}$ than by intakes of other nutrients (RR 1.19; $95 \%$ CI 0.95 , 1.49; $P$ value, test for trend $=0 \cdot 10)$. No associations were found between the consumption of red meat (beef and pork), poultry and the risk of type 2 diabetes.

When the diabetes cases diagnosed during the first 5 years of follow-up were excluded ( $n$ 417) from the analyses, the results between the consumption of total meat as well as of specific types of meats and the risk of type 2 diabetes did not change. For example, the risk of type 2 diabetes (adjusted for risk factors related to diabetes) was 1.52 (95\% CI 1.14, 2.01; $P$ value, test for trend $<0.001$ ) for the highest quintile of total meat consumption, and was 1.46 (95\% CI 1.11 , $1.92 ; P$ value, test for trend $=0 \cdot 01$ ) for processed meat.

The associations between the consumption of total meat, processed meat and the risk of type 2 diabetes were not modified by BMI ( $P$ value, test for interaction $\geq 0 \cdot 30$ ).

\section{Discussion}

In the present cohort study of Finnish male smokers followed up to 12 years, the multivariate relative risk (e.g. BMI and energy) of type 2 diabetes was $50 \%$ higher for the highest 
Table 2. Risk of diabetes by quintiles of meat consumption among 25943 men in the Alpha-Tocopherol, Beta-Carotene Cancer Prevention study, Finland, 1985-97

(Relative risks (RR) and $95 \%$ confidence intervals)

\begin{tabular}{|c|c|c|c|c|c|c|c|c|c|c|}
\hline & \multicolumn{9}{|c|}{ Quintiles of meat consumption } & \multirow[b]{3}{*}{$P$ for trend } \\
\hline & \multirow{2}{*}{$\begin{array}{c}1 \\
\mathrm{RR}\end{array}$} & \multicolumn{2}{|c|}{2} & \multicolumn{2}{|c|}{3} & \multicolumn{2}{|c|}{4} & \multicolumn{2}{|c|}{5} & \\
\hline & & $\mathrm{RR}$ & $95 \% \mathrm{Cl}$ & $\mathrm{RR}$ & $95 \% \mathrm{Cl}$ & $\mathrm{RR}$ & $95 \% \mathrm{Cl}$ & $\mathrm{RR}$ & $95 \% \mathrm{Cl}$ & \\
\hline \multicolumn{11}{|l|}{ Total meat } \\
\hline Median (g/d) & 79 & 111 & & 139 & & 174 & & 244 & & \\
\hline Cases $(n)$ & 181 & 192 & & 225 & & 220 & & 280 & & \\
\hline Age-adjusted* & 1.00 & 1.03 & $0.83,1.26$ & $1 \cdot 19$ & $0.97,1.46$ & $1 \cdot 15$ & $0.94,1.41$ & 1.50 & $1.23,1.82$ & $<0.001$ \\
\hline Multivariate† & 1.00 & 1.05 & $0.85,1.29$ & $1 \cdot 22$ & $1.00,1.50$ & $1 \cdot 19$ & $0.96,1.47$ & 1.45 & $1 \cdot 16,1 \cdot 81$ & $<0.001$ \\
\hline Multivariate $\ddagger$ & 1.00 & 1.06 & $0.85,1.31$ & 1.24 & $1.00,1.53$ & 1.22 & $0.98,1.52$ & 1.50 & $1 \cdot 19,1.89$ & $<0.001$ \\
\hline \multicolumn{11}{|l|}{ Red meat } \\
\hline Median (g/d) & 33 & 47 & & 60 & & 76 & & 106 & & \\
\hline Cases $(n)$ & 189 & 217 & & 241 & & 219 & & 232 & & \\
\hline Age-adjusted* & 1.00 & 1.12 & $0.92,1.37$ & 1.22 & $1.00,1.48$ & 1.08 & $0.89,1.32$ & 1.14 & $0.93,1.39$ & 0.33 \\
\hline Multivariate† & 1.00 & 1.23 & $1.00,1.50$ & 1.31 & $1.07,1.59$ & $1 \cdot 16$ & $0.94,1.42$ & 1.19 & $0.97,1.47$ & 0.23 \\
\hline Multivariateł & 1.00 & 1.24 & $1.01,1.52$ & $1 \cdot 33$ & $1.08,1.63$ & $1 \cdot 18$ & $0.95,1.46$ & 1.22 & $0.97,1.53$ & 0.21 \\
\hline \multicolumn{11}{|l|}{ Beef } \\
\hline Median (g/d) & 6 & 14 & & 20 & & 29 & & 47 & & \\
\hline Cases $(n)$ & 189 & 221 & & 222 & & 217 & & 249 & & \\
\hline Age-adjusted* & 1.00 & 1.14 & $0.94,1.39$ & $1 \cdot 12$ & $0.92,1.37$ & 1.09 & $0.89,1.33$ & 1.23 & $1.01,1.50$ & 0.09 \\
\hline Multivariate† & 1.00 & $1 \cdot 13$ & $0.93,1.38$ & $1 \cdot 19$ & $0.97,1.45$ & $1 \cdot 12$ & $0.91,1.36$ & 1.23 & $1.01,1.50$ & 0.08 \\
\hline Multivariate $\ddagger$ & 1.00 & 1.13 & $0.92,1.37$ & $1 \cdot 19$ & $0.98,1.46$ & $1 \cdot 11$ & $0.91,1.37$ & 1.22 & $0.99,1.50$ & $0 \cdot 10$ \\
\hline \multicolumn{11}{|l|}{ Pork } \\
\hline Median (g/d) & 19 & 29 & & 37 & & 47 & & 66 & & \\
\hline Cases $(n)$ & 216 & 200 & & 224 & & 250 & & 208 & & \\
\hline Age-adjusted* & 1.00 & 0.89 & $0.74,1.09$ & 0.99 & $0.82,1.20$ & 1.10 & $0.91,1.33$ & 0.90 & $0.74,1.09$ & 0.99 \\
\hline Multivariate† & 1.00 & 0.94 & $0.77,1.14$ & 1.04 & $0.85,1.26$ & 1.14 & $0.94,1.38$ & 0.96 & $0.78,1.18$ & 0.57 \\
\hline Multivariate $\ddagger$ & 1.00 & 0.94 & $0.77,1.15$ & 1.04 & $0.85,1.27$ & 1.15 & $0.95,1.41$ & 0.97 & $0.78,1.20$ & 0.50 \\
\hline \multicolumn{11}{|l|}{ Processed meat } \\
\hline Median (g/d) & 22 & 42 & & 60 & & 86 & & 142 & & \\
\hline Cases $(n)$ & 176 & 186 & & 236 & & 243 & & 257 & & \\
\hline Age-adjusted* & 1.00 & 1.04 & $0.84,1.28$ & 1.32 & $1.08,1.61$ & 1.35 & $1.10,1.64$ & 1.46 & $1 \cdot 20,1 \cdot 77$ & $<0.001$ \\
\hline Multivariate $\dagger$ & 1.00 & 1.04 & $0.84,1.29$ & 1.26 & $1.03,1.55$ & $1 \cdot 19$ & $0.97,1.46$ & 1.35 & $1.09,1.68$ & $<0.001$ \\
\hline Multivariate $\ddagger$ & 1.00 & 1.04 & $0.84,1.29$ & 1.26 & $1.03,1.54$ & $1 \cdot 19$ & $0.96,1.46$ & 1.37 & $1 \cdot 11,1 \cdot 71$ & $<0.001$ \\
\hline \multicolumn{11}{|l|}{ Poultry } \\
\hline Median (g/d) & 0 & 8 & & 14 & & 17 & & 32 & & \\
\hline Cases $(n)$ & 366 & 174 & & 179 & & 165 & & 214 & & \\
\hline Age-adjusted* & 1.00 & 0.86 & $0.72,1.04$ & 1.05 & $0.88,1.27$ & 0.89 & $0.74,1.07$ & $1 \cdot 15$ & $0.96,1.36$ & 0.25 \\
\hline Multivariate† & 1.00 & 0.92 & $0.77,1 \cdot 11$ & 1.00 & $0.83,1.20$ & 0.92 & $0.76,1 \cdot 11$ & 1.04 & $0.87,1.23$ & 0.88 \\
\hline Multivariate $\ddagger$ & 1.00 & 0.90 & $0.75,1.09$ & 0.98 & $0.82,1 \cdot 18$ & 0.89 & $0.74,1.08$ & 1.01 & $0.85,1.21$ & 0.88 \\
\hline
\end{tabular}

${ }^{*}$ Adjusted for age and intervention group.

†Adjusted further for BMI, number of cigarettes smoked daily, smoking years, systolic blood pressure, diastolic blood pressure, serum total cholesterol, serum HDL- cholesterol, leisure-time physical activity and intakes of alcohol and energy.

$\ddagger$ Adjusted further for consumption of fruits, vegetables, rye, milk and coffee.

quintile of total meat consumption compared with the lowest quintile. Especially, high consumption of processed meat was associated with a $35 \%$ increased risk of type 2 diabetes compared with the diet low in processed meat (median consumption on average 22 v. $142 \mathrm{~g}$ ), also after adjustments for environmental and dietary factors. The consumption of red meat (beef and pork) and poultry was not associated with the risk of type 2 diabetes.

A 20-30\% higher risk of type 2 diabetes has been observed for the highest category of frequent red meat consumption compared with the lowest category in the Nurses' Health Study and in the Women's Health Study ${ }^{(7,8)}$, and a 40-90\% higher risk has been observed for the consumption of processed meat at least five times a week compared with the consumption less than once a week in the Nurses' Health Study and in the Health Professionals Follow-up Study ${ }^{(6,15)}$. Longterm adherence to a diet that included at least weekly meat consumption was associated with a $74 \%$ increased risk of diabetes compared with a vegetarian diet ${ }^{(16)}$. In a large Chinese female cohort with a very low intake of meat, the consumption of processed meat ( $>$ once a month $v$. never) was also slightly associated with the risk of type 2 diabetes $^{(9)}$, especially among the obese women $\left(B M I \geq 30 \mathrm{~kg} / \mathrm{m}^{2}\right.$ ) whose risk of type 2 diabetes was 3.5-fold higher compared with the women with normal weight. A relatively small cohort study among Japanese-Brazilians found that high meat consumption was related to the risk of the metabolic syndrome ${ }^{(17)}$. The result, however, attenuated when the model was adjusted for the intake of SFA. Furthermore, two cross-sectional studies have found contradictory results ${ }^{(18,19)}$. The present study is the first European cohort study on this issue. Furthermore, our male population was totally different from the previous male cohorts, the well-educated health professionals ${ }^{(15)}$ and the participants in the Adventist Health Study. Our population, in general, included lowly educated smokers (about 10\% smokers in the Health Professionals Follow-up Study) whose 
coffee and alcohol consumption was high (on average 18 and $610 \mathrm{~g} / \mathrm{d}$, respectively). In the present study, the range of meat consumption was especially high, between 79 and $244 \mathrm{~g} / \mathrm{d}$ (median in the lowest and highest quintiles) including mainly red meat and sausages. The results of the present study, however, confirmed the previous findings that the high consumption of processed meat seemed to be a risk factor of type 2 diabetes more than the high total meat consumption. The results were not modified by BMI.

The mechanisms related to the positive associations between red meat or processed meat consumption and type 2 diabetes are unclear. It has been suggested that the associations observed are mediated through high intake of fat, $\mathrm{SFA}^{(2)}$, protein $^{(8)}$, haeme $\mathrm{Fe}^{(20,21)}$, preservatives used in processed meat (such as nitrates and nitrite) ${ }^{(22)}$, heterocyclic amines and polycyclic aromatic hydrocarbons formed in meat through high heating practice ${ }^{(23,24)}$, or glycation end products formed in meat and high-fat products through heating and processing ${ }^{(25)}$. These dietary factors have been found to affect insulin resistance ${ }^{(26)}$, oxidative stress ${ }^{(27)}$, inflammation $^{(28)}$ and toxically pancreatic cells ${ }^{(23)}$. In our data, the attenuation of RR was explained more by the intakes of $\mathrm{Na}$ than by intakes of SFA, protein, cholesterol, haeme $\mathrm{Fe}, \mathrm{Mg}$, nitrate, energy, alcohol, fruits, vegetables, rye, milk or coffee. The other factors related to preservation or cooking meat at high temperature could not be included in our analyses. The effect of nitrite was difficult to assess because of the very high correlation between nitrite and total meat intakes $(r$ 0.82). On the other hand, high meat consumption may be a biomarker for a general lifestyle related to high risk of type 2 diabetes.

A strength of the present study was the prospective cohort design, which minimises recall and selection biases. We also had large amounts and ranges of the consumption of total meat and the specific types of meats. Although we were able to adjust for main non-dietary risk factors of type 2 diabetes, we cannot entirely rule out the possibility of residual or unmeasured confounding.

The ATBC study included only male smokers, which should be noted when the results are extrapolated to women or non-smokers. Furthermore, the drug reimbursement register was not able to separate the types of diabetes (type 1 and type 2 diabetes). We assumed, however, that the new diabetes cases in the present study had type 2 diabetes based on the age (50-69 years) of the participants at baseline. We were able to identify only patients receiving medication for the treatment of diabetes, not individuals treating their disease with dietary changes. This will attenuate our estimates between meat consumption and diabetes incidence towards unity. Furthermore, we had a single assessment of diet by a FFQ at baseline, and were not able to investigate changes in meat consumption during the follow-up. This may have contributed to the misclassification of exposure, which will also attenuate the observed associations.

Maintenance of normal weight, avoidance of sedentary behaviour and smoking, moderate alcohol consumption and healthy diet are the most potential preventive factors against type 2 diabetes $^{(29)}$. The present findings confirmed that a diet poor in meat, especially processed meat, may also help to prevent type 2 diabetes.

\section{Acknowledgements}

S. M. prepared the first draft of the manuscript; J. K. analysed the data; all authors designed the study, interpreted analyses, refined the subsequent drafts and provided consultation on the final draft. None of the authors had any conflicts of interest. The ATBC study was supported by US Public Health Service contracts N01-CN-45 165, N01-RC-45 035 and N01-RC-37004 from the National Cancer Institute, National Institutes of Health and the Department of Health and Human Services.

The present study has been given Clinical Trials. gov ID no. NCT00342992.

\section{References}

1. King H, Aubert RE \& Herman WH (1998) Global burden of diabetes, 1995-2025. Diabetes Care 21, 1414-1431.

2. Parillo M \& Riccardi G (2004) Diet composition and the risk of type 2 diabetes: epidemiological and clinical evidence. $\mathrm{Br} J$ Nutr 92, 7-19.

3. Vazques G, Duval S, Jacobs DR Jr, et al. (2007) Comparison of body mass index, waist circumference, and waist/hip ratio in predicting incident diabetes: a meta-analysis. Epidemiol Rev 29, 115-128.

4. Pan XR, Li GW, Hu YH, et al. (1997) Effects of diet and exercise in preventing NIDDM in people with impaired glucose tolerance. The Da Qing IGT and Diabetes Study. Diabetes Care 20, 537-544.

5. Tuomilehto J, Lindström J, Eriksson JG, et al. (2001) Prevention of type 2 diabetes mellitus by changes in lifestyle among subjects with impaired glucose tolerance. $N$ Engl J Med 344, $1343-1350$.

6. van Dam RM, Willett WC, Rimm EB, et al. (2002) Dietary fat and meat intake in relation to risk of type 2 diabetes in men. Diabetes Care 25, 417-424.

7. Fung TT, Schulze M, Manson JE, et al. (2004) Dietary patterns, meat intake, and the risk of type 2 diabetes in women. Arch Intern Med 164, 2235-2240.

8. Song Y, Manson JE, Buring JE, et al. (2004) A prospective study of red meat consumption and type 2 diabetes in middleaged and elderly women. Diabetes Care 27, 2108-2115.

9. Villegas R, Shu XO, Gao YT, et al. (2006) The association of meat intake and the risk of type 2 diabetes may be modified by body weight. Int J Med Sci 3, 152-159.

10. The ATBC Cancer Prevention Study Group (1994) The alpha-tocopherol, beta-carotene lung cancer prevention study: design, methods, participant characteristics, and compliance. Ann Epidemiol 4, 1-10.

11. The Alpha-Tocopherol Beta-Carotene Cancer Prevention Study Group (1994) The effect of vitamin E and beta carotene on the incidence of lung cancer and other cancers in male smokers. $N$ Engl J Med 330, 1029-1035.

12. Pietinen P, Hartman AM, Haapa E, et al. (1988) Reproducibility and validity of dietary assessment instruments. I. A self-administered food use questionnaire with a portion size picture booklet. Am J Epidemiol 128, 655-666.

13. Willett W (1998) Nutritional Epidemiology, 2nd ed. Oxford: Oxford University Press.

14. R Development Core Team (2008) R: A Language and Environment for Statistical Computing. Vienna: R Foundation for Statistical Computing.

15. Schulze MB, Manson JE, Willett WC, et al. (2003) Processed meat intake and incidence of type 2 diabetes in younger and middle-aged women. Diabetologia 46, 1465-1473.

16. Vang A, Singh PN, Lee JW, et al. (2008) Meats, processed meats, obesity, weight gain and occurrence of diabetes among 
adults: findings from adventist health studies. Ann Nutr Metab 52, 96-104.

17. Damiao R, Castro TG, Cardoso MA, et al. (2006) Dietary intakes associated with metabolic syndrome in a cohort of Japanese ancestry. Br J Nutr 96, 532-538.

18. Mennen LI, Lafay L, Feskens EJM, et al. (2000) Possible protective effect of bread and dairy products on the risk of the metabolic syndrome. Nutr Res 20, 335-347.

19. Azadbakht L \& Esmaillzadeh A (2009) Red meat intake is associated with metabolic syndrome and the plasma C-reactive protein concentration in women. J Nutr 139, 335-339.

20. Tuomainen TP, Nyyssönen K, Salonen R, et al. (1997) Body iron stores are associated with serum insulin and blood glucose concentrations population study in 1013 eastern Finnish men. Diabetes Care 20, 426-428.

21. Jiang R, Manson JE, Meigs JB, et al. (2004) Body iron stores in relation to risk of type 2 diabetes in apparently healthy women. JAMA 291, 711-717.

22. Portha B, Giroix MH, Cros JC, et al. (1980) Diabetogenic effect of $N$-nitrosomethylurea and $N$-nitrosomethylurethane in adult rat. Ann Nutr Aliment 34, 1143-1151.
23. LeDoux SP, Woodley SE, Patton NJ, et al. (1986) Mechanisms of nitrosourea-induced $\beta$-cell damage: alterations in DNA. Diabetes 35, 866-872.

24. Lijinsky W (1999) N-nitroso compounds in the diet. Mutat Res 443, 129-138

25. Peppa M, Goldberg T, Cai W, et al. (2002) Glycotoxins: a missing link in the relationship of dietary fat and meat intake in relation to risk of type 2 diabetes in men. Diabetes Care 25, $1896-1899$

26. Hofmann SM, Dong HJ, Li Z, et al. (2002) Improved insulin sensitivity is associated with restricted intake of dietary glycoxidation products in the $\mathrm{db} / \mathrm{db}$ mouse. Diabetes 51, 2082-2089.

27. Cai W, Gao QD, Zhu L, et al. (2002) Oxidative stress inducing carbonyl compounds from common foods: novel mediators of cellular dysfunction. Mol Med 8, 337-346.

28. Biondi-Zoccai GG, Abbate A, Liuzzo G, et al. (2003) Atherosclerosis, imflammation, and diabetes. J Am Coll Cardiol 41, 1071-1077.

29. Schulze MB \& Hu FB (2005) Primary prevention of diabetes: what can be done and how much can be prevented. Annu Rev Public Health 26, 445-467. 